\title{
Reassessment of an Origin of the Radio Structure of J1420-0545
}

\author{
M. Jamrozy ${ }^{1}$ (D), J. Machalski ${ }^{1}$, B. Nikiel-Wroczyński ${ }^{1}$, and H. T. Intema ${ }^{2}$ (D) \\ ${ }^{1}$ Astronomical Observatory, Jagiellonian University, ul. Orla 171, 30-244 Kraków, Poland; jamrozy@oa.uj.edu.pl, machalsk@oa.uj.edu.pl, iwan@oa.uj.edu.pl \\ ${ }^{2}$ Leiden Observatory, Leiden University, Niels Borhweg 2, NL-2333CA, Leiden, The Netherlands; intema@strw.leidenuniv.nl \\ Received 2017 April 21; revised 2017 September 25; accepted 2017 October 3; published 2017 November 13
}

\begin{abstract}
In this paper, we test the possibility that the structure of the largest radio galaxy J1420-0545 may have been formed by restarted rather than primary jet activity. This hypothesis was motivated by the unusual morphological properties of the source consisting of two edge-brightened, narrow, highly collinear, and symmetric lobes, thus suggesting an almost ballistic propagation of powerful jets into a particularly low-density external medium. New observations made with the VLA together with the currently available GLEAM and TGSS ADR1 data releases allow the detection of an excess emission at low frequencies. An extracted part $(88 \mathrm{MHz}-200 \mathrm{MHz})$ of the spectrum of the emission is fitted with the DYNAGE model, giving a forecast for the environmental conditions and the energetic requirements for the presumed old cocoon related to a preceding epoch of jet activity.
\end{abstract}

Key words: galaxies: active - galaxies: individual (J1420-0545) - galaxies: jets

\section{Introduction}

The largest ( $\gtrsim 1 \mathrm{Mpc}$ ) radio galaxies are believed to have evolved from smaller sources; however, it is almost certain that most of the observed small sources will never evolve into giant sizes. An evolutionary scheme for the general population of radio sources, from the smallest gigahertz peaked spectrum (GPS), through the compact steep-spectrum (CSS) ones, until the FRII/FRI (Fanaroff \& Riley 1974) structures, was discussed by Snellen et al. (2000). Some, rather theoretical, predictions concerning this scheme was presented by Kaiser \& Best (2007). Most known, and well-studied, giant radio galaxies (GRGs) usually are sources of FRII-type morphology. There are a number of factors considered underlying the gigantic size of some radio sources: (i) high jet power, (ii) sufficiently low density of the ambient medium into which the jet propagates, and (iii) the lifetime of jet activity. An interesting idea is given in the paper by Subrahmanyan et al. (1996) presenting the research on several GRGs, where it was postulated that the giants could become very large after several jet activity cycles. Indeed, among the so-called double-double radio galaxies (DDRGs), there are a number of GRGs.

The largest known radio galaxy, J1420-0545 (located at R.A.(J2000.0) $14^{\mathrm{h}} 20^{\mathrm{m}} 23^{\mathrm{s}} .8$ and decl.(J2000.0) $\left.-05^{\circ} 45^{\prime} 28^{\prime \prime} 8\right)$, having an angular size of $17 ! 4$, was discovered by Machalski et al. (2008). Its weak radio core coincides with a parent galaxy at a spectroscopic redshift of 0.3067 (presented in their paper). The source's double structure with a total projected linear size of $4.7 \mathrm{Mpc}$ is extremely slim. This structure, consisting of two opposite narrow lobes (with an axial ratio, i.e., the ratio of the length to width of the whole radio structure, of about 12, measured according to a prescription given by Leahy \& Williams 1984), is highly collinear and symmetric; the arm ratio is only 1.08, and the misalignment angle is 1.3 . These properties are very characteristic of the inner lobes of known radio galaxies with a double-double structure (cf. Schoenmakers et al. 2000).

There is no doubt that understanding the temporal evolution of extragalactic radio sources involves accessing not only the jet parameters (its kinetic power, speed, lifetime) and the properties of the ambient medium into which the lobes evolve, but also the complex duty cycle of jet activity. Current studies indicate that this activity can be intermittent, or at least highly modulated on different timescales (for a review, see, e.g., Saikia \& Jamrozy 2009). There is direct observational evidence that the restarted jets usually do not propagate through the undisturbed intergalactic medium (IGM), but instead within the environment substantially modified by the passage of the outflow during the previous stage of jet activity (Kaiser et al. 2000; Safouris et al. 2008). This may affect the observed properties of the newly formed lobes.

In a previous paper (Machalski et al. 2011; hereafter MJSK), the authors considered the possibility that the apparent double structure of J1420-0545 might be formed by restarted, rather than primary, jet activity. The motivation for the performed analysis was the high axial ratio of its lobes, which is the same order as-or even higher than-such ratios for the inner lobes in all well-studied DDRGs, as well as the extremely low density of the external environment, considerably young age of the structure, and the resulting very high speed of the jet head's propagation $(\sim 0.2 c$, in contrast to $0.03 c-0.05 c$ for the inner lobes in typical DDRGs; Kaiser et al. 2000; MJSK)—where all of these properties were derived using the fitting procedure of the analytical model of the source's evolution. The question was whether such a low density would be due to the unique location of J1420-0545 in a large void region or due to some previous jet activity epoch causing a substantial rarefaction of the IGM. In MJSK, a model of the evolution of that hypothetical primary structure (lobes) was assumed, and its expected luminosity and radio spectrum were estimated. The current paper extends the observational effort to detect the relic's emission.

These new Karl G. Jansky Very Large Array (hereafter VLA) observations of J1420-0545, the relevant low-frequency data taken from the already available GaLactic and Extragalactic All-sky MWA Survey (GLEAM; Wayth et al. 2015; Hurley-Walker et al. 2017) and the Giant Metrewave Radio Telescope (GMRT) Sky Survey (TGSS) First Alternative Data Release (ADR1; Intema et al. 2017), as well as the data reduction are presented in Section 2. Fortunately, the new data allow the detection of an excess emission at low frequencies, which possibly originated from some old population of 
relativistic particles that may be related to the presumed cocoon (lobes), i.e., a relic of a previous episode of nuclear activity. The relevant data reduction and the modeling task applied are described in Section 3. A discussion of the results and final conclusions are summarized in Section 4. As in previous papers, the distance, linear size, and luminosity of the analyzed source are calculated for the $\Lambda \mathrm{CDM}$ cosmology with $\Omega_{\mathrm{m}}=0.27, \Omega_{\Lambda}=0.73$, and $H_{0}=71 \mathrm{~km} \mathrm{~s}^{-1} \mathrm{Mpc}^{-1}$.

\section{New Radio Data}

\subsection{VLA Observations}

The VLA observations were carried out in the C-array configuration. The $P$-band data were collected as part of project 14B-156 (PI: M. Jamrozy), consisting of three scheduling blocks (performed in 2014 December 17, 23, and 30), each $\sim 1790$ s long (excluding time spent for setting the antennas, and for the amplitude and phase calibrations, the total time on the target was about $2000 \mathrm{~s}$ ). Each of these blocks used the same instrumental setup: the central frequency was set to $352 \mathrm{MHz}$ and the total bandwidth was $256 \mathrm{MHz}$ divided into 16 subbands, each consisting of 128 narrow channels. Such a scheme was chosen to avoid multichannel RFI contamination. The strong and unresolved source 3C 295 was used as the primary calibrator, while 3C 298 was taken as the phase calibrator. The final synthesized beam was 63 ! $7 \times 48$ !" 8 at a P.A. of $-26^{\circ} .5$.

For the data reduction, all of the VLA data sets were imported to the Common Astronomy Software Applications package (CASA) and underwent a standard procedure, as outlined in the "P-band basic data reduction" guide. ${ }^{3}$ The most important processing steps involved ionospheric correction, flagging bad data points (both automatic and finetouch manual), and the calibration of flux density (according to Scaife \& Heald 2012), delay, bandpass, gain, and instrumental polarization. All of these steps were done on the primary calibrator, and the corrections were then transferred to the target field. All three blocks were concatenated and deconvolved using the CLEAN algorithm. The resulting map was used to start a self-calibration loop, but as there was no improvement-either qualitative or quantitative-no matter the scheme (phase only, amplitude and phase), it was not carried on.

\subsection{GLEAM and TGSS ADRI Data}

The (GLEAM) survey, performed with the Murchison Widefield Array (MWA; Lonsdale et al. 2009; Tingay et al. 2013) and overlying the entire sky south of decl. $+25^{\circ}$, is described in detail by Wayth et al. (2015). The survey covers the radio frequency range between 72 and $231 \mathrm{MHz}$, divided into five bands (with the central frequencies of 87.5, 118.5, 154.5, 185.0, and $215.7 \mathrm{MHz}$ ), providing nearly continuous coverage but avoiding the band around $137 \mathrm{MHz}$, which is contaminated by satellite interference. The angular resolution of the survey is $2 ! 5 \times 2 ! 2 \sec \left(\delta+26^{\circ} .7\right)$ at $154 \mathrm{MHz}$. The output of the first year of GLEAM comprises both postage stamp FITS maps and the radio source catalog published by Hurley-Walker et al. (2017). A set of 20 images with the bandwidth of 7.7 MHz and a wideband image within $170-231 \mathrm{MHz}$ (with a resolution of $\sim 2^{\prime}$ )

\footnotetext{
3 https://casaguides.nrao.edu/index.php/0313-192_P-band_basic_data_ reduction-CASA4.6
}

is provided. In addition, a set of three stacked maps covering the ranges $72-103,103-134$, and $139-170 \mathrm{MHz}$ is also available. All of the data (images and catalog) are publicly accessible on the MWA Telescope website. ${ }^{4}$

The TGSS ADR1 survey was conducted at $150 \mathrm{MHz}$ between 2010 and 2012. All of its archival raw data were reprocessed with a fully automated pipeline based on the Source Peeling and Atmospheric Modeling (SPAM) package (Intema et al. 2009; Intema 2014), which includes a directiondependent calibration, modeling, and tasks correcting mainly ionospheric dispersive phase delay. TGSS ADR1 (Intema et al. 2017) includes continuum Stokes $I$ images of the radio sky north of decl. $-53^{\circ}$, with the resolution of $25^{\prime \prime} \times 25^{\prime \prime}$ north of decl. $19^{\circ}$ and $25^{\prime \prime} \times 25^{\prime \prime} \sec \left(\delta-19^{\circ}\right)$ south of decl. $19^{\circ}$. The noise level is usually below $5 \mathrm{mJy} \mathrm{beam}^{-1}$. Intema et al. (2017) described the details of the data processing and publicly available products (images and catalog). ${ }^{5}$ However, it is worth noting that the TGSS ADR1 data are compiled excluding the short visibilities within a $0.2 \mathrm{k} \lambda$ distance of the $(u, v)$-plane coverage of the GMRT baselines. Its impact on the modeling procedure is explored in Section 3.1

\section{Data Reduction and Modeling}

\subsection{New Radio Maps and the Spectrum of J1420-0545}

The new low-frequency maps of J1420-0545 obtained from the above data are compiled in Figure 1, and the instrumental characteristics of all radio maps included in this paper are collected in Table 1.

Further steps in the data reduction consisted of the (i) correction of the flux densities of the narrow opposite lobes for the emission of the compact background source that is confusing the northern lobe and is clearly visible on the high-resolution maps in MJSK (their Figure 2), (ii) determination of the corrected radio spectrum of these lobes, and (iii) separation of their low-frequency emission from that detected in the GLEAM survey. In the first step, the spectrum of the confusing source given in MJSK (column 7 in their Table 4) was extrapolated to the frequency of $148 \mathrm{MHz}$, with the slope of 0.58 arising from its flux densities at 329 and $619 \mathrm{MHz}$ and used to correct the flux densities measured at the VLA and TGSS ADR1 maps where its contribution is not resolved (the confusing source position on the VLA and TGSS ADR1 maps is marked in Figure 1). In order to determine the spectrum of the slim lobes' structure at frequencies below $\sim 150 \mathrm{MHz}$, a synchrotron model was fitted to those corrected flux densities but excluding the GLEAM data. This is done in order to separate the excess emission in the low-resolution GLEAM frequencies relative to the compact emission detected with the highresolution observations at frequencies above $\sim 150 \mathrm{MHz}$. Because of the significant steepness of this spectrum at frequencies above $1400 \mathrm{MHz}$, the JP (Jaffe \& Perola 1973) model was used to evaluate the flux densities of these lobes at the GLEAM frequencies. The fitted models, including or excluding the TGSS ADR1 data point, appear almost identical-the flux densities expected at the GLEAM frequencies differ by less than $1 \mathrm{mJy}$. Finally, the flux densities determined this way were subtracted from those resulting from the GLEAM survey. The latter ones are the flux densities of the entire radio

\footnotetext{
4 http://www.mwatelescope.org/science/gleam-survey

5 http://tgssadr.strw.leidenuniv.nl/
} 


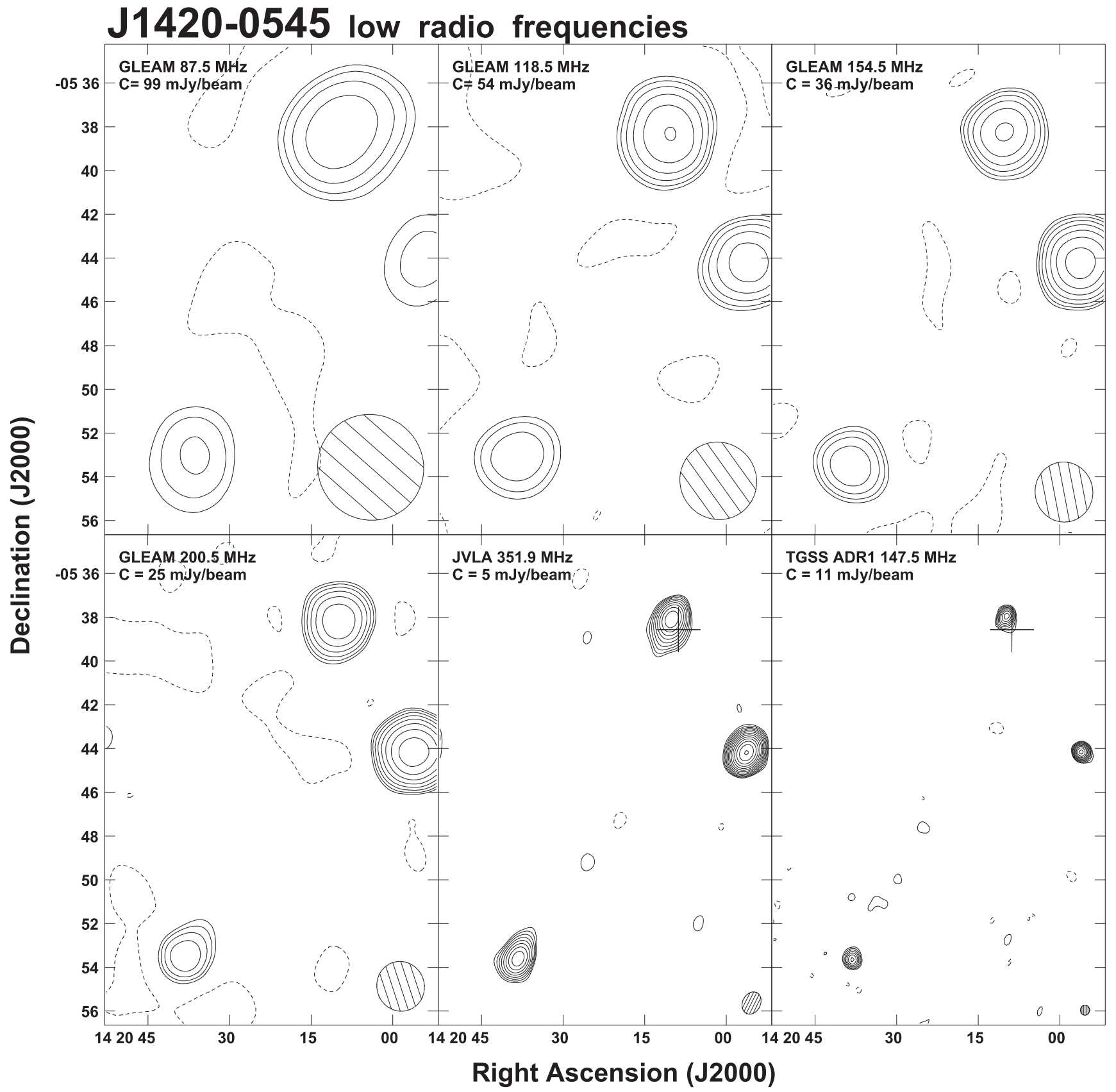

Figure 1. Radio maps of J1420-0545 (the northern and southern lobes) observed at six different frequencies: $87.5 \mathrm{MHz}, 118.5 \mathrm{MHz}, 154.5 \mathrm{MHz}$, and $200.5 \mathrm{MHz}$ from GLEAM, as well as at $147.5 \mathrm{MHz}$ from TGSS ADR1 and at $351.9 \mathrm{MHz}$ from VLA. The contours, spaced in factors of $\sqrt{2}$ in brightness, are plotted starting with the value $\mathrm{C}\left(\simeq 3 \times\right.$ the rms noise in the relevant map; cf. Table 1) given in each panel in units of mJy beam ${ }^{-1}$. The cross marks the position of the background confusing source. The sizes of the beams are indicated by the hatched circles/ellipses in the bottom-right corner of each image.

Table 1

Instrumental Characteristics of the Presented Radio Maps

\begin{tabular}{lcccc}
\hline \hline $\begin{array}{l}\text { Telescope } \\
\text { Survey }\end{array}$ & $\begin{array}{c}\text { Frequency } \\
\nu(\mathrm{MHz})\end{array}$ & $\begin{array}{c}\text { Beam Size } \\
(\operatorname{arcsec})\end{array}$ & $\begin{array}{c}\text { P.A. } \\
(\mathrm{deg})\end{array}$ & $\begin{array}{c}\text { rms Noise } \\
\left(\mathrm{mJy} \mathrm{beam}^{-1}\right)\end{array}$ \\
\hline MWA/ & 87.5 & $294 \times 287$ & 48.7 & 32.9 \\
GLEAM & 118.5 & $215 \times 209$ & 35.6 & 17.8 \\
& 154.5 & $165 \times 158$ & 10.9 & 12.3 \\
GMRT/ & 200.5 & $137 \times 130$ & 18.1 & 8.5 \\
TGSS ADR1 & 147.5 & $27.6 \times 25.0$ & 0.0 & 3.8 \\
VLA & 351.9 & $63.7 \times 48.8$ & -26.5 & 1.7 \\
\hline
\end{tabular}

structure within the four frequency bands [72-103], [103-134], [139-170], and [170-231] MHz, and were derived by averaging their values provided in the Hurley-Walker et al. (2017) catalog with our Gaussian-model fits performed on the FITS maps with the Astronomical Image Processing System AIPS task JMFIT.

All of the above radio data are collected in Table 2 . Columns 2 and 3 give the above averaged GLEAM flux densities, and those derived from the TGSS ADR1 and VLA maps, respectively. A similar procedure is performed for the error analysis. Column 4 gives the flux densities of the confusing source given in MJSK and supplemented with their estimates at the frequencies of GLEAM and TGSS ADR1, while 
Table 2

New Observed Flux Densities of the Entire Structure of J1420-0545 (Columns 2 and 3) and the Archival Data for its Slim Lobes and the Background Source Confusing the Northern Lobe (Columns 4 and 5 with the Relevant Notes Appended under the Table)

\begin{tabular}{|c|c|c|c|c|c|c|}
\hline \multirow{4}{*}{$\begin{array}{l}\nu[\mathrm{MHz}] \\
(1)\end{array}$} & \multicolumn{2}{|c|}{ Entire Structure } & \multirow{2}{*}{$\begin{array}{l}\text { N-conf. } \\
\text { Source }\end{array}$} & \multirow{2}{*}{$\begin{array}{c}\text { Slim Lobes } \\
\text { Corrected }\end{array}$} & \multirow{2}{*}{$\begin{array}{l}\text { Residual } \\
\text { Flux }\end{array}$} & \multirow{2}{*}{$\begin{array}{c}\text { Model } \\
\text { Fit }\end{array}$} \\
\hline & GLEAM & TGSS/VLA & & & & \\
\hline & $S_{\nu}(\mathrm{mJy})$ & $S_{\nu}(\mathrm{mJy})$ & $S_{\nu}(\mathrm{mJy})$ & $S_{\nu}(\mathrm{mJy})$ & $S_{\nu}(\mathrm{mJy})$ & $S_{\nu}(\mathrm{mJy})$ \\
\hline & (2) & (3) & (4) & (5) & (6) & (7) \\
\hline 87.7 & $681 \pm 110$ & $\ldots$ & $\ldots$ & $441 \pm 13^{c}$ & $240 \pm 40$ & 264.9 \\
\hline 118.4 & $540 \pm 68$ & $\ldots$ & $\ldots$ & $382 \pm 5^{c}$ & $158 \pm 20$ & 151.6 \\
\hline 147.5 & $\ldots$ & $369 \pm 19$ & $26^{\mathrm{b}}$ & $343 \pm 17$ & $\ldots$ & $\ldots$ \\
\hline 154.3 & $420 \pm 40$ & $\ldots$ & $\ldots$ & $335 \pm 4^{\mathrm{c}}$ & $85 \pm 9$ & 84.3 \\
\hline 351.9 & $\ldots$ & $232 \pm 13$ & $15.4^{\mathrm{b}}$ & $217 \pm 11$ & $\ldots$ & $\ldots$ \\
\hline 617.3 & $\ldots$ & $\ldots$ & $11.1 \pm 1.8^{\mathrm{a}}$ & $153 \pm 11^{\mathrm{a}}$ & $\ldots$ & $\ldots$ \\
\hline 1400.0 & $\ldots$ & $\cdots$ & $5.1 \pm 0.7^{\mathrm{a}}$ & $90 \pm 8^{\mathrm{a}}$ & $\cdots$ & $\cdots$ \\
\hline 4860.1 & $\cdots$ & $\ldots$ & $2.7 \pm 0.4^{\mathrm{a}}$ & $22.7 \pm 2.3^{\mathrm{a}}$ & $\ldots$ & $\ldots$ \\
\hline
\end{tabular}

Notes. Columns 6 and 7 give the low-frequency residual flux densities interpreted as the presumed relic emission of a former cocoon and the DYNAGE fit to this relic, respectively.

${ }^{a}$ Flux densities from MJSK.

${ }^{\mathrm{b}}$ Extrapolated flux densities.

${ }^{\mathrm{c}}$ Flux densities from the JP model fit shown in Figure 2.

column 5 gives the flux densities of the narrow opposite lobes dominating the high-frequency maps in MJSK. The values in brackets are estimated from the synchrotron model fit mentioned before. The flux densities in column 6 are related to the residual emission at low frequencies, i.e., they show the difference between the entries in columns 2 and 5, while column 7 indicates the DYNAGE model fit to these values described in Section 3. The spectra resulting from the above data are presented in Figure 2.

The "residual" flux densities, i.e., the difference between their values detected in the GLEAM survey and the flux densities estimated for the apparent lobes of J1420-0545, are significant, suggesting an excess of the low-frequency emission with a very steep spectral index ranging from $\alpha=1.9 \pm 0.03$ at $88 \mathrm{MHz}$ to $\alpha=2.7 \pm 0.05$ at $200 \mathrm{MHz}$. Such a spectrum resembles well-known spectra that are a superposition of two components: steep-spectrum extended lobes and a flatspectrum compact radio core, i.e., where the emission of a bright core strongly flattens their high-frequency slope. In the case of J1420-0545, such a bend-up appears at low frequencies. In order to check whether this might be caused by the incompatible flux-density scales applied in the sky surveys involved, we check the spectrum of the neighboring compact source MRC 1419-053 (PKS B1419-053). Its spectrum, shown in Figure 3, includes the flux-density points collected from all of the surveys involved, i.e., GLEAM, TGSS ADR1, and VLA, as well as points from other databases. All data points that originated from the above surveys and supplemented with the $74 \mathrm{MHz}$ flux density from the VLA Low-Frequency Sky Survey Redux (VLSSr) catalog (Lane et al. 2014) indicate a consistency of their calibration scales. In this context, we note that the flux densities of the latter source are overestimated in the $365 \mathrm{MHz}$ Texas survey (TXS; Douglas et al. 1996) and in the $408 \mathrm{MHz}$ Molonglo Reference Catalog (MRC; Large et al. 1981). Thus, we cannot exclude the possibility that the observed low-frequency emission excess may be related to the hypothetical cocoon that formed during a previous episode of jet activity precluding the one actually observed, i.e., the scenario supposed and considered in MJSK.

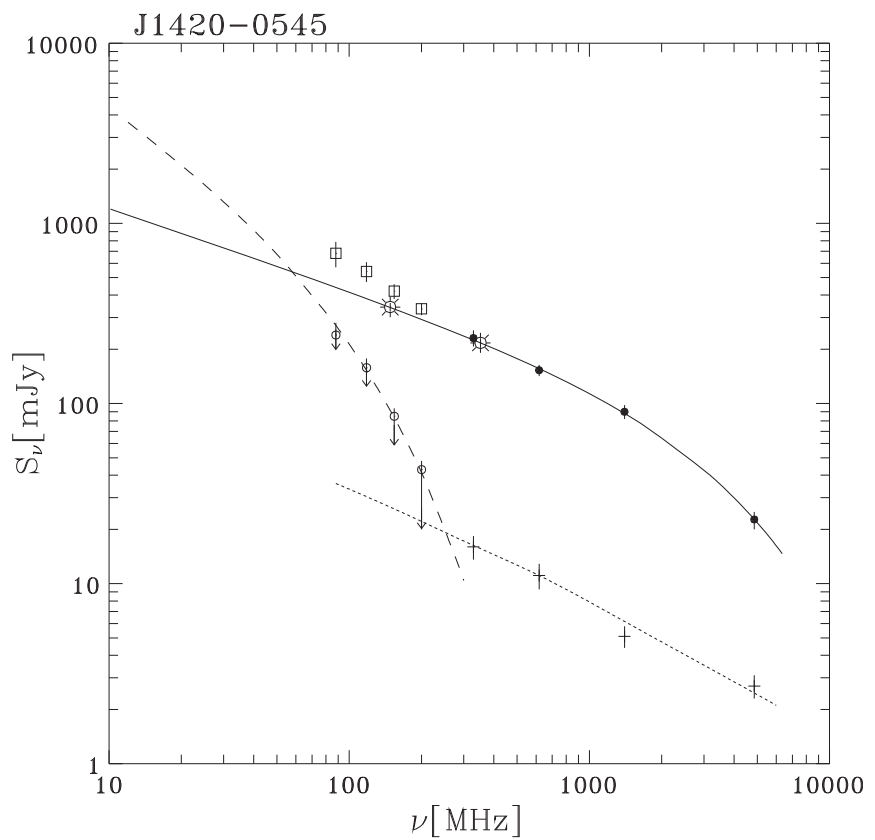

Figure 2. Radio spectra of the apparent narrow lobes of J1420-0545 fitted with the JP model (the solid line), the faint source confusing the northern lobe (the dotted line), and the DYNAGE model fit to the residual data points that originated within the low-frequency range of the spectrum from the GLEAM survey (the dashed line). Different symbols mark the flux-density data points collected in Table 2: open squares-average GLEAM data (column 2), crosses-confusing source (column 4), large crossed open circles and small dots-corrected TGSS ADR1 /VLA and original MJSK data, respectively (column 5), and small unfilled circles-residual data (column 6). Explanations for the arrows connected to them are given in Section 4.

In that paper, it was completely hypothetical. However, the new observations presented here change the situation; the emission excess at the low frequencies cannot be ignored. Therefore, we undertake a further attempt to fit a dynamical model to these residual flux-density points. 


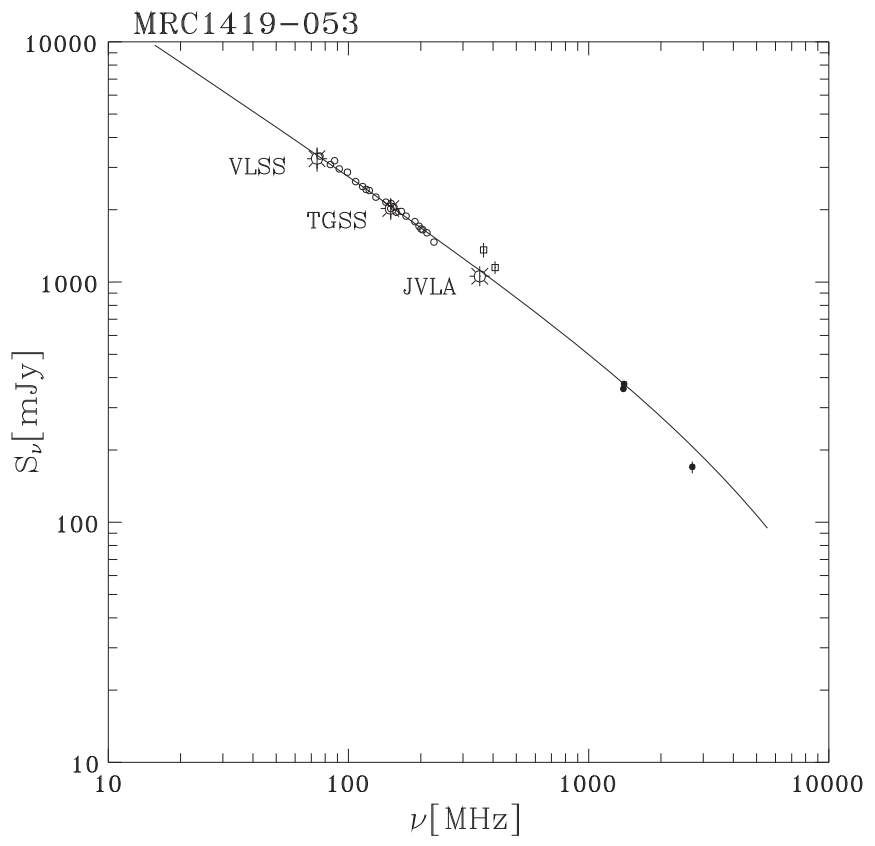

Figure 3. Radio spectrum of the neighboring source MRC 1419-053 used to control the consistency of the flux-density scales at low frequencies. All of the GLEAM channel data are marked by small unfilled circles. The VLSSr, TGSS ADR1, and VLA flux densities are shown by large crossed unfilled circles, while small dots indicate the FIRST (Becker et al. 1995), NVSS (Condon et al. 1998), and PKS (Wright \& Otrupcek 1990) fluxes. The TXS and MRC data are marked by small unfilled squares. The solid line shows the JP model fitted to the data points.

\subsection{Modeling Procedure}

Similarly to previous works (e.g., Machalski et al. 2008, $2011,2016)$, the modeling procedure performed in this paper uses the DYNAGE algorithm of Machalski et al. (2007). This numerical code allows the determination, for a given set of observables, of the four main parameters of the dynamical model, the jet power $Q_{\mathrm{j}}$, the density of the external gaseous medium near the radio core $\rho_{0}$, the age of the lobes' structure $t$, and the initial exponent of the electron energy distribution injected by the jets into the lobes $p=1+2 \alpha_{\text {inj }}$, where $\alpha_{\text {inj }}$ is the injection spectral index, which characterizes the slope of the spectrum at a very young age of the radio source. However, the apparent slope of the four residual flux-density points in Figure 2 strongly suggests an emission of some old population of relativistic electrons, e.g., a relic emission typical for "dying" (old) sources, i.e., those whose activity of their nuclei was terminated or fell down to such a low level that a jet outflow would be inefficient or disappear completely (cf. Komissarov \& Gubanov 1994; Murgia et al. 2011). For this reason, an extension of the DYNAGE code (the dynamical model with terminated jet activity, KDA EXT), described by Kuligowska (2017), was applied. The extension of the DYNAGE code is based on the division of the cocoon's temporal evolution into two periods: the time of the jet activity until its termination $\left(t_{\mathrm{j}}\right)$ and that from the terminated activity until the actual age of the source, $t$. The evolution during the first period is described in detail in Machalski et al. (2007). A further evolution after $t_{\mathrm{j}}$, proposed by Kuligowska (2017), relying on modified expressions for the adiabatic expansion of the cocoon, its pressure, and integrated radio power, is summarized in the Appendix.
Table 3

Model Input Parameters for the Presumed Old Cocoon

\begin{tabular}{|c|c|c|}
\hline $\begin{array}{l}\text { Parameter } \\
\text { (1) }\end{array}$ & $\begin{array}{l}\text { Symbol } \\
\text { (2) }\end{array}$ & $\begin{array}{l}\text { Value } \\
\text { (3) }\end{array}$ \\
\hline \multicolumn{3}{|l|}{ Set: } \\
\hline Linear size & $D[\mathrm{kpc}]$ & 4690 \\
\hline Axial ratio & $R_{T}$ & 6.0 \\
\hline Jet power & $Q_{\mathrm{j}}\left[10^{45} \mathrm{erg} \mathrm{s}^{-1}\right]$ & 3.9 \\
\hline Adiabatic index of the cocoon material & $\Gamma_{\mathrm{c}}$ & $5 / 3$ \\
\hline Adiabatic index of the ambient medium & $\Gamma_{\mathrm{x}}$ & $5 / 3$ \\
\hline Adiabatic index of the magnetic field & $\Gamma_{\mathrm{B}}$ & $4 / 3$ \\
\hline Minimum electron Lorentz factor (injected) & $\gamma_{\min }$ & 1 \\
\hline Maximum electron Lorentz factor (injected) & $\gamma_{\max }$ & $10^{7}$ \\
\hline $\begin{array}{l}\text { Core radius of the ambient density } \\
\text { distribution }\end{array}$ & $a_{0}[\mathrm{kpc}]$ & 10 \\
\hline Slope of the ambient density distribution & $\beta$ & $3 / 2$ \\
\hline $\begin{array}{l}\text { Thermal particles within the cocoon } \\
\text { Observed: }\end{array}$ & $k^{\prime}$ & 10 \\
\hline $\begin{array}{l}\text { Luminosity spectral densities at a number } \\
\text { of observing frequencies, } i=1,2,3,4\end{array}$ & $P_{\nu_{i}}$ & Note \\
\hline $\begin{array}{l}\text { Fit: } \\
\text { Jet power }(!)\end{array}$ & $Q_{\mathrm{j}}\left[10^{45} \mathrm{erg} \mathrm{s}^{-1}\right]$ & 4.5 \\
\hline Central density & $\rho_{0}\left[10^{-26} \mathrm{~g} \mathrm{~cm}^{-3}\right]$ & 3.9 \\
\hline Injection spectral index & $\alpha_{\mathrm{inj}}$ & 0.65 \\
\hline Cocoon age & $t[\mathrm{Myr}]$ & 315 \\
\hline Jet activity duration & $t_{\mathrm{j}}[\mathrm{Myr}]$ & 110 \\
\hline External density & $\rho_{\mathrm{D} / 2}\left[10^{-29} \mathrm{~g} \mathrm{~cm}^{-3}\right]$ & 1.1 \\
\hline Cocoon pressure & $p_{\mathrm{c}}\left[10^{-13}\right.$ dyn $\left.\mathrm{cm}^{-2}\right]$ & 3.0 \\
\hline Goodness of the fit & $\chi_{\text {red }}^{2}$ & 0.54 \\
\hline
\end{tabular}

Note. Relevant luminosities are calculated using the flux densities given in column 6 of Table $2($ !) — see the text.

In the first step of the modeling, we repeated the calculations of MJSK using the new data for the narrow lobes (the entries in column 5 of Table 2), and realized that the earlier models for the "Primary" or "Inner" origin of the structure are not changed appreciably. In the second step, we fit the KDA EXT model to the entries in column 6 of Table 2. Because of the lack of data at frequencies below $329 \mathrm{MHz}$ in MJSK, the authors had to assume values for two of the four model parameters predictable by the model fit, i.e., the values of $Q_{\mathrm{j}}$ and $\rho_{0}$. In this paper, we only assume the $Q_{\mathrm{j}}$ value, equating it to the value fitted in the "Inner" case solution. The other required assumptions, kept identical as in MJSK, are summarized in Table 3. The last eight lines in Table 3 show the fit results. Note that the best-fit value of $Q_{\mathrm{j}}$ is 1.14 times higher than the initially assumed value. Such an increase was necessary to obtain a better normalization of the modeled spectrum without changing its shape. Likewise, the central density in the present solution is comparable to that in MJSK. Further aspects of the fit are discussed in the next section.

\section{Discussion and Conclusions}

Since GLEAM's restoring beam sizes are much larger than the corresponding beams in the high-frequency maps in both MJSK and this paper, the residual flux densities (in column 6 of Table 2) do not account for an additional flux which the GLEAM observations might detect from eventual steep-spectrum sources not seen in the higher-frequency bands. An upper limit on this additional flux would be determined by fitting a JP model to the sum of entries (columns 4 and 5) and the entries in 
column 3. As the result, we realize that the residual flux densities (column 6) might decrease to $\sim 85 \%$ at $88 \mathrm{MHz}$ until $\sim 50 \%$ at $200 \mathrm{MHz}$, as shown by the arrows in Figure 2. In such a situation, the radio spectrum of the presumed old cocoon would steepen even more than that shown by the dashed line in Figure 2. Keeping the above in mind, we might expect that a relevant DYNAGE model will predict a little lower jet power and a higher age than their values given in Table 3 . In this context, it is worth emphasizing that the radiative ages determined for the relic radio sources have never exceeded $300 \mathrm{Myr}$ (cf. Slee et al. 2001; Murgia et al. 2011). Therefore, this is very likely that a low surface brightness of the old cocoon may be hard to detect, and its brightest regions are only visible with GLEAM.

A significant difference between the presented model of the presumed old cocoon and the "Outer" solution in MJSK (the last column in their Table 6) is the high $\alpha_{\text {inj }}$ value of 0.65. Such values usually characterize the steep or very steep spectra of sources observed at high redshifts. This is expected due to the evidence for the kinetic temperature and density of the IGM evolution with redshift. Therefore, at higher redshifts, the radio jet works against the higher pressure of the denser ambient medium (cf. Athreya \& Kapahi 1998). In such circumstances, the propagation speed of the jet's head, as well as the bulk flow behind this head, would slow down. Consequently, the acceleration process would lead to a steeper value of $p$ in the energy distribution. However, the very large size and uncommon propagation speed of J1420-0545 (resulting from its relatively young age determined from the model) testify against a dense environment. The analysis of Kuligowska (2017) shows how much the radio spectrum will steepen in relation to its initial slope when a period of quiescence is comparable to the lifetime of the jet activity, even if $\alpha_{\text {inj }}$ is not very high.

Another difference between the presented model and the "Outer" model in MJSK is the fitted age of the old cocoon, 315 Myr versus $178 \mathrm{Myr}$. However, both solutions were based on arbitrary assumptions about the jet power during the primary nuclear activity, $Q_{\mathrm{j} \text {,out }} \simeq Q_{\mathrm{j} \text {,inn }}$ and $Q_{\mathrm{j} \text {,out }} \simeq 3 \times Q_{\mathrm{j} \text {,inn }}$, respectively. Such assumptions were necessary considering a very short part of the radio spectrum that is suspected of being a relic, as well as simply invisible in MJSK. Therefore, one can expect that several quite different models may fit the data points in column 6 of Table 2). However, the difference between the ages of the presumed old cocoon derived within those solutions, which are comparable to the fitted time lags $t-t_{\mathrm{j}}(\sim 110 \mathrm{Myr}$ and $\sim 106 \mathrm{Myr}$, respectively), suggests an uncertainty of the ages not larger than $50 \%$ of their fitted values. Further observations at frequencies much lower than $70 \mathrm{MHz}$ would help constrain the variety of such models.

On the other hand, the models presented here and in MJSK provide similar very low densities of the gas surrounding the hypothetical primary lobes of J1420-0545 $\left(\rho_{\text {out }} \sim 10^{-29} \mathrm{~g} \mathrm{~cm}^{-3}\right)$. This result, together with the density of the new cocoon related to the observed slim lobes $\left(\rho_{\text {inn }} \sim 2 \times 10^{-31} \mathrm{~g} \mathrm{~cm}^{-3}\right)$ predicted in MJSK and the fact that the latter value is consistent with the mean density of the baryonic matter in the universe, implies that the extensive discussion of these issues in MJSK (in their Section 4.4) still remains valid.

Some doubts concerning the long time lag between subsequent jet activities implied by the model derived in this paper, and uncertainty whether such a low-density environment caused by a primary phase of activity would remain in place for the current phase, may arise. This was already considered and extensively discussed by Kaiser et al. (2000). They argued that replacement of the old cocoon material by the surrounding external medium (due to the buoyancy effect and/or a possible pressure gradient within the cocoon) proceeds at the sound speed in this medium. Therefore, although these effects undoubtedly depend on its kinetic temperature, the entrainment of gaseous material is a slow process, and Kaiser et al. (2000), p. 389 concluded: “...it seems unlikely that the environments of the inner sources of the DDRGs are created by the replacement of the old cocoon material by the denser IGM." In the case of J1420-0545, proper X-ray observations and eventual detection of radial surface brightness and gas density profiles in the vicinity of its host galaxy would be crucial for discriminating between the two possible origins of the observed radio structure: (i) repeated periods of jet activity or (ii) a unique (primary) episode of the jet activity appearing in a deep void of the IGM at the outskirts of a filamentary Warm-Hot IGM (WHIM). For a comprehensive review concerning cosmic voids, see, e.g., van de Weygaert \& Platen (2011).

To sum up, in this paper, we test the possibility that the structure of the largest radio galaxy, J1420-0545, discovered by Machalski et al. (2008), may be formed by restarted, rather than primary, jet activity. As mentioned in Section 1, this hypothesis was motivated by the unusual morphological properties of the source, suggesting an almost ballistic propagation of powerful jets in a particularly low-density ambient medium. Numerical simulations of the development of jets in a pre-existing cocoon of synchrotron emission have been presented by Clarke \& Burns (1991). They suggest that the supersonic propagation of a restarted jet in the old cocoon can excite a weak bow shock immediately ahead of this jet. However, this scenario seems to be inconsistent with observations of Mpc-scale DDRGs, which show the inner double structures as edge-brightened lobes rather than nearballistic jets. In this context, Clarke (1997) considered the possibility that a very large Mach number may force the bow shock to hug the jet along its length, so that the emission from both the bow shock and the restarted jet would together form a narrow inner structure. The obtained results and the final conclusions are as follows.

1. New observations of J1420-0545 conducted with the VLA and the data provided from TGSS ADR1 extend the low-frequency spectrum of its two narrow lobes. Repeated calculations in MJSK using these supplementary data show that the earlier dynamical models for these lobes are not changed appreciably. Different model fits performed for the observed radio structure imply a relatively young age of the source $\sim 35 \mathrm{Myr}$, relatively high expansion velocity $\sim 0.2 c$, and large kinetic power $\sim 4 \times 10^{45} \mathrm{erg} \mathrm{s}^{-1}$, and confirm a particularly lowdensity environment $\lesssim 10^{-29} \mathrm{~g} \mathrm{~cm}^{-3}$.

2. The emission detected with the GLEAM survey seems to support the hypothesis about the presence of an old cocoon expected in the framework of the jet intermittency scenario. It is intriguing that the brightest regions in the GLEAM maps overlap the narrow lobes presumed to be formed by restarted jet activity. Their emission is likely contaminated by an old population of relativistic electrons from a previous episode of nuclear activity. The lack of detectable emission between these regions means that, even if it exists, its radio surface brightness is too low to be discerned from the cosmic background. 
3. In spite of the above, we conclude that the dilemma of whether the extremely low density of the J1420-0545 environment is due to previous jet activity or to its unique location in a large void region of the galaxy distribution is still not settled. Perhaps, future Low Frequency Array (LOFAR) observations in the low-frequency band $(15 \mathrm{MHz}-70 \mathrm{MHz})$ may help in investigating the presence of a hypothetical outer cocoon in this source; however, the southern declination of the target might cause a problem.

This scientific work makes use of the Murchison Radioastronomy Observatory, operated by CSIRO. We acknowledge the Wajarri Yamatji people as the traditional owners of the observatory site. Support for the operation of the MWA is provided by the Australian Government (NCRIS), under contract to Curtin University administered by Astronomy Australia Limited. We acknowledge the Pawsey Supercomputing Centre, which is supported by the Western Australian and Australian Governments. The authors acknowledge the National Radio Astronomy Observatory, which is a facility of the National Science Foundation operated under cooperative agreement by Associated Universities, Inc., for observing time, as well as the staff of the GMRT that made these observations possible. GMRT is run by the National Centre for Radio Astrophysics of the Tata Institute of Fundamental Research. We also thank Dr. Thomas Franzen for explanations concerning the GLEAM data and the anonymous referee for useful suggestions that helped improve the paper. J.M. and M.J. were supported in part by the Polish National Science Centre (NSC) grant DEC-2013/09/B/ ST9/00599.

\section{Appendix}

The density distribution of the ambient gaseous medium, $\rho_{\mathrm{a}}$ (identical for both periods: jet activity and after its termination), is $\rho_{\mathrm{a}}(r)=\rho_{0}\left(r / a_{0}\right)^{-\beta}$, where $\rho_{0}$ is the central density of the radio core with radius $a_{0}$, and $\beta$ is the exponent of the density profile.

The total length of the jet $r_{\mathrm{j}}$ arising from energy conservation conditions, i.e., approximately one-half of the source's linear size at the time of termination, $D\left(t_{\mathrm{j}}\right)$, is

$$
r_{\mathrm{j}}\left(t_{\mathrm{j}}\right)=c_{1}\left(\frac{Q_{\mathrm{j}}}{\rho_{0} a_{0}^{\beta}}\right)^{1 /(5-\beta)} t_{\mathrm{j}}^{3 /(5-\beta)} \approx \frac{D\left(t_{\mathrm{j}}\right)}{2},
$$

where $Q_{\mathrm{j}}$ is the jet's power and $c_{1}$ is a constant dependent on the ratio of the jet head's pressure and the uniform cocoon pressure, $\mathcal{P}_{\mathrm{hc}}$, as a function of the cocoon's axial ratio $R_{\mathrm{T}}$. Note that an explanation of all parameters describing a source and its analytical model is given in Table 3 .

The source's length at its actual age, $t \geqslant t_{\mathrm{j}}$, is given by

$$
D\left(t, t_{\mathrm{j}}\right)=D\left(t_{\mathrm{j}}\right)\left(\frac{t}{t_{\mathrm{j}}}\right)^{c_{4}}
$$

where $c_{4}=\frac{2\left(\Gamma_{\mathrm{c}}+1\right)}{\Gamma_{\mathrm{c}}\left(7+3 \Gamma_{\mathrm{c}}-2 \beta\right)}$.

The cocoon's pressure after the jet's activity switches off is

$$
p_{\mathrm{c}}\left(t>t_{\mathrm{j}}\right)=p_{\mathrm{c}}\left(t_{\mathrm{j}}\right)\left(\frac{t}{t_{\mathrm{j}}}\right)^{-3 \Gamma_{\mathrm{c}} c_{4}},
$$

where the uniform cocoon pressure during the jet's activity, $p_{\mathrm{c}}\left(t_{\mathrm{j}}\right)$, is the same as that given by Kaiser \& Alexander. (1997, their Equation (20)).

The analytical formula for the total ratio power, $P_{\nu}$, of a source (its cocoon) at a given frequency is written as the sum of two integrals. The first integral gives the power calculated until the time $t_{\mathrm{j}}$, while the second one adds the power of the emission from $t_{\mathrm{j}}$ until the actual age of the source, $t$,

$$
P_{\nu}(t)= \begin{cases}P_{\nu}\left(t_{\min }, t_{\mathrm{j}}\right)+P_{\nu}\left(t_{\mathrm{j}}, t\right) & \text { for } t_{\mathrm{j}}>t_{\mathrm{min}} \\ P_{\nu}\left(t_{\min }, t\right) & \text { for } t_{\mathrm{j}} \leqslant t_{\mathrm{min}}\end{cases}
$$

where $t_{\min }$ is the injection time of the particles with the largest Lorentz factors permissible by the model. In the above equation, the first term corresponds to the integral given by Kaiser et al. (1997, their Equation (16)). The second term is given by

$$
P_{\nu}\left(t_{\mathrm{j}}, t\right)=\frac{\sigma_{T} c}{6 \pi \nu} \frac{r}{r+1} Q_{\mathrm{j}} \mathcal{P}_{\mathrm{hc}}^{\left(1-\Gamma_{\mathrm{c}}\right) / \Gamma_{\mathrm{c}}} \int_{t_{0}}^{t} H\left(t_{\mathrm{i}}\right) G\left(t_{\mathrm{i}}\right) d t_{\mathrm{i}},
$$

where $t_{0}=t_{\min }$ if $t_{\mathrm{j}} \leqslant t_{\min }$ and $t_{0}=t_{\mathrm{j}}$ if $t_{\mathrm{j}}>t_{\min }$,

$$
H\left(t_{\mathrm{i}}\right)=n_{0}\left(t_{\mathrm{i}}\right) \frac{\gamma^{3-p} t_{\mathrm{i}}^{a_{1} / 3(p-2)}}{\left[t^{-a_{1} / 3}-a_{2}\left(t, t_{\mathrm{i}}\right) \gamma\right]^{2-p}}\left(\frac{t}{t_{\mathrm{i}}}\right)^{-a_{1}\left(1 / 3+\Gamma_{\mathrm{B}}\right)},
$$

$G\left(t_{\mathrm{i}}\right)$

$$
=\frac{\int_{0}^{1} F_{\mathrm{JP}}(x) x^{-p}(1-x)^{p-2} d x}{\int_{0}^{1} F_{\mathrm{CI}}(x) x^{-(p+1)}\left[1-(1-x)^{p-2}\right] d x+\int_{1}^{\infty} F_{\mathrm{JP}}(x) x^{-(p+1)} d x},
$$

where the energy distribution of the injected particles is $n(\gamma)=n_{0} \gamma^{-p}$ and $p=1+2 \alpha_{\mathrm{inj}}$. The functions $F_{\mathrm{JP}}=$ $\nu / \nu_{\mathrm{br}, \mathrm{JP}} / x^{2}$ and $F_{\mathrm{CI}}=\nu / \nu_{\mathrm{br}, \mathrm{Cl}} / x^{2}$, where the frequency breaks in the radio spectrum, are

$$
\begin{gathered}
\nu_{\mathrm{br}, \mathrm{CI}}=\frac{c_{\nu}}{c_{\epsilon}^{2}} \frac{B}{\left(B^{2}+B_{\mathrm{iC}}^{2}\right)^{2} t^{2}}, \text { and } \\
\nu_{\mathrm{br}, \mathrm{PP}}=\frac{c_{\nu}}{c_{\epsilon}^{2}} \frac{B}{\left(\frac{2}{3} B^{2}+B_{\mathrm{iC}}^{2}\right)^{2}\left(t-t_{\mathrm{j}}\right)^{2}},
\end{gathered}
$$

$c_{\nu}$ and $c_{\epsilon}$ are physical constants (for details, see Pacholczyk 1970 ), and $B$ and $B_{\mathrm{iC}}$ are the strengths of the magnetic field in the source (cocoon) and the equivalent field associated with the inverse Compton scattering of the CMB photons, respectively.

\section{ORCID iDs}

M. Jamrozy (i) https://orcid.org/0000-0002-0870-7778

H. T. Intema (i) https://orcid.org/0000-0002-5880-2730

\section{References}

Athreya, R. M., \& Kapahi, V. K. 1998, JApA, 19, 63

Becker, R. H., White, R. L., \& Helfand, D. J. 1995, ApJ, 450, 559

Clarke, D. A. 1997, in ASP Conf. Ser. 123, Computational Astrophysics, ed. D. A. Clarke \& M. J. West (San Francisco, CA: ASP), 255

Clarke, D. A., \& Burns, J. O. 1991, ApJ, 369, 308

Condon, J. J., Cotton, W. D., Greisen, E. W., et al. 1998, AJ, 115, 1693

Douglas, J. N., Bash, F. N., Bozyan, F. A., Torrence, G. W., \& Wolfe, C. 1996, AJ, 111,1945

Fanaroff, B. L., \& Riley, J. M. 1974, MNRAS, 167, 31

Hurley-Walker, N., Callingham, J. R., Hancock, P. J., Frazen, T. M. O., et al. 2017, MNRAS, 464, 1146

Intema, H. T. 2014, SPAM, Astrophysics Source Code Library, ascl:1408.006 
Intema, H. T., Jagannathan, P., Mooley, K. P., \& Frail, D. A. 2017, A\&A, 598, 78

Intema, H. T., van der Tol, S., Cotton, W. D., et al. 2009, A\&A, 501, 1185

Jaffe, W. J., \& Perola, G. C. 1973, A\&A, 26, 423

Kaiser, C. R., \& Alexander., P. 1997, MNRAS, 286, 215

Kaiser, C. R., \& Best, P. N. 2007, MNRAS, 381, 1548

Kaiser, C. R., Dennett-Thorpe, J., \& Alexander, P. 1997, MNRAS, 292, 723

Kaiser, C. R., Schoenmakers, A. P., \& Röttgering, H. J. A. 2000, MNRAS, 315,381

Komissarov, S. S., \& Gubanov, A. G. 1994, A\&A, 285, 27

Kuligowska, E. 2017, A\&A, 598, 93

Lane, W. M., Cotton, W. D., van Velzen, S., Clarke, T. E., et al. 2014, MNRAS, 440, 327

Large, M. I., Mills, B. Y., Little, A. G., Crawford, D. F., \& Sutton, J. M. 1981, MNRAS, 194, 693

Leahy, J. P., \& Williams, A. G. 1984, MNRAS, 210, 929

Lonsdale, C. J., Cappallo, R. J., Morales, M. F., et al. 2009, IEEEP, 97, 1497

Machalski, J., Chyży, K. T., Stawarz, Ł., \& Kozieł, D. 2007, A\&A, 462, 43

Machalski, J., Jamrozy, M., Stawarz, Ł., \& Kozieł-Wierzbowska, D. 2011, ApJ, 740, 58

Machalski, J., Jamrozy, M., Stawarz, L., \& Weżgowiec, M. 2016, A\&A, 595,46
Machalski, J., Kozieł-Wierzbowska, D., Jamrozy, M., \& Saikia, D. J. 2008, ApJ, 679, 149

Murgia, M., Parma, P., Mack, K.-H., de Ruiter, H. R., et al. 2011, A\&A, 526, 148

Pacholczyk, A. G. 1970, Radio Astrophysics (San Francisco, CA: Freeman)

Safouris, V., Subrahmanyan, R., Bicknell, G. V., \& Saripalli, L. 2008, MNRAS, 385, 2117

Saikia, D. J., \& Jamrozy, M. 2009, BASI, 37, 63

Scaife, A. M., \& Heald, G. H. 2012, MNRAS, 423, 30

Schoenmakers, A. P., de Bruyn, A. G., Röttgering, H. J. A., van der Laan, H., \& Kaiser, C. R. 2000, MNRAS, 315, 371

Slee, O. B., Roy, A. L., Murgia, M., Andernach, H., \& Ehle, M. 2001, AJ, 122,1172

Snellen, I. A. G., Schilizzi, R. T., Miley, G. K., de Bruyn, A. G., et al. 2000, MNRAS, 319, 445

Subrahmanyan, R., Saripalli, L., \& Hunstead, R. W. 1996, MNRAS, 279, 257

Tingay, S. J., Goeke, R., Bowman, J. D., Emrich, D., et al. 2013, PASA, 30, 7

van de Weygaert, R., \& Platen, E. 2011, IJMPS, 1, 41

Wayth, R. B., Lenc, E., Bell, M. E., Callingham, R. J., et al. 2015, PASA, 32, 25

Wright, A., \& Otrupcek, R. 1990, Parkes Catalogue (Epping: Australia Telescope National Facility) 